\title{
Identification of Cardiac Myosin Peptides Capable of Inducing Autoimmune Myocarditis in BALB/c Mice
}

\author{
Christian L. Pummerer, ${ }^{\star}$ Kerstin Luze, ${ }^{\star}$ Gerhard GrässI, ${ }^{\star}$ Kurt Bachmaier, ${ }^{\star}$ Felix Offner,; Sarah K. Burrell, \\ Thomas J. Zamborelli, $\|$ Josef M. Penninger, ${ }^{\S}$ and Nikolaus Neu* \\ *Department of Pediatrics and ${ }^{\ddagger}$ Department of Pathology, University of Innsbruck, Medical School, A-6020 Innsbruck, Austria; ${ }^{\circledR}$ Amgen \\ Institute, Ontario Cancer Institute, Department of Medical Biophysics and Immunology, University of Toronto, Toronto, Ontario, Canada

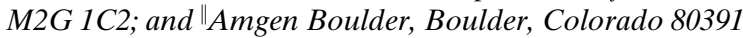

\begin{abstract}
Immunization with cardiac myosin induces $\mathrm{T}$ cell-mediated myocarditis in genetically predisposed mice and serves as a model for autoimmune heart disease. This study was undertaken to identify pathogenic epitopes on the myosin molecule. Our approach was based on the comparison of the pathogenicity between cardiac $(\alpha-)$ myosin and soleus muscle ( $\beta-)$ myosin. We show that $\alpha$-myosin is the immunodominant isoform and induces myocarditis at high severity and prevalence whereas $\beta$-myosin induces little disease. Therefore the immunodominant epitopes of $\alpha$-myosin must reside in regions of different amino acid sequence between $\alpha$ - and $\beta$-myosin isoforms. Cardiac myosin peptides corresponding to these regions of difference were synthesized and tested for their ability to induce inflammatory heart disease. Three pathogenic peptides were identified. One peptide that is located in the head portion of the molecule induced severe myocarditis, whereas two others that reside in the rod portion possessed only minor pathogenicity. The identification of pathogenic epitopes on the cardiac myosin molecule will allow detailed studies on the recognition of this antigen by the immune system and might be used to downmodulate ongoing heart disease. (J. Clin. Invest. 1996. 97:2057-2062.) Key words: cardiology • autoimmunity $\bullet$ myositis $\bullet$ isoforms - epitopes
\end{abstract}

\section{Introduction}

Postviral myocarditis is a serious disorder and often results in dilated cardiomyopathy. Aberrant immune responses have been implicated by several clinical and experimental studies (1-4). Based on the hypothesis that certain forms of this disease are mediated by an autoimmune response to intracellular proteins released after virus-mediated myocyte damage, we have previously established a murine model where myocarditis is inducible by purified cardiac myosin (5). This model serves as a virus-free system to investigate the pathological mechanisms acting in postinfectious autoimmune heart disease. Car-

Address correspondence to Dr. Nikolaus Neu, Department of Pediatrics, University of Innsbruck, Medical School, Anichstrasse 35, A-6020 Innsbruck, Austria. Phone: 512-504-3600; FAX: 512-5043484.

Received for publication 17 November 1995 and accepted in revised form 14 February 1996.

J. Clin. Invest.

(C) The American Society for Clinical Investigation, Inc.

0021-9738/96/05/2057/06 \$2.00

Volume 97, Number 9, May 1996, 2057-2062 diac myosin-induced myocarditis has been shown to be $\mathrm{CD}^{+}$ $\mathrm{T}$ cell-mediated and strictly dependent on MHC class II expression $(6,7)$. One of the most important questions is which epitope(s) on the cardiac myosin molecule is (are) crucial for the induction of the disease. Also, it is not clear whether the autoreactive $\mathrm{T}$ cells recognize other myosin isoforms in addition to the cardiac isoform.

The major obstacle to identifying the relevant epitopes is the great length of the myosin molecule which consists of two 200-kD heavy chains and two pairs of low molecular mass light chains. In vertebrates, the myosin heavy chains are encoded by a multigenic family and comprise various isoforms which are under tissue-specific regulation. The only myosin isoform found in the cardiac tissue of young adult rodents is the $\alpha$-isoform, whereas the soleus muscle, in addition to type II A- and II B-myosin, mainly contains the $\beta$-isoform but no $\alpha$-myosin. The contractile apparatus of the other skeletal muscles, the socalled fast white skeletal muscles, exclusively contains the II A- and II B-myosin isoforms at various ratios (for reviews see references 8-11). As previously shown, in contrast to the cardiac $(\alpha-)$ myosin, these two isoforms induce neither myositis nor myocarditis (5).

However, the pathogenicity of the $\beta$-myosin isoform has not been tested. In contrast to other skeletal muscle myosin isoforms the $\beta$-myosin differs from the $\alpha$-myosin only by circumscribed amino acid regions. Here we demonstrate that $\beta$-myosin is a weak inducer of inflammatory heart disease as compared with the immunodominant $\alpha$-myosin isoform. Based on this finding, we synthesized cardiac $(\alpha-)$ myosin peptides corresponding to the regions of difference between the $\alpha$ - and $\beta$-myosin isoforms which allowed the identification of three epitopes with major and minor pathogenicity.

\section{Methods}

Experimental animals. BALB/cJ HanHim mice and Black Dolly rats were obtained from the Institute for Laboratory Animals (Himberg, Austria). The BALB/c J HanHim mice were originally derived from the Jackson Laboratories (Bar Harbor, ME) and were further maintained by brother/sister mating and constant genetic control at the Animal Facilities of Hannover, Germany, and subsequently at Himberg, Austria.

Antigen preparation. Cardiac myosin was chromatographically purified from 4-wk-old Black Dolly rats and soleus muscle myosin was chromatographically purified from 12 -wk-old Black Dolly rats as described previously (12). These ages were selected because cardiac tissues of young rats exclusively contain $\alpha$-myosin, whereas those from older animals also contain minor amounts of $\beta$-myosin (10). Conversely, the percentage of $\beta$-myosin in the soleus muscle increases with the age of the animals (13). Murine cardiac and soleus myosin was prepared from pooled hearts and soleus muscles obtained from various strains of adult mice. Due to the small amount of tissue available, murine soleus muscle myosin was purified according to the 


\begin{tabular}{lcc}
\hline \multicolumn{1}{c}{ Immunization } & Myocarditis & Soleus muscle myositis* \\
\cline { 2 - 3 } Murine $\alpha$-myosin (cardiac myosin) & Prevalence $^{\ddagger}$ (severity \pm SD) & \\
Murine $\beta$-myosin (soleus muscle myosin) & $16 / 25(3.6 \pm 0.5)$ & $0 / 25$ \\
Rat $\alpha$-myosin (cardiac myosin) & $2 / 18(1 \pm 0)$ & $1 / 18$ \\
Rat $\beta$-myosin (soleus muscle myosin) & $13 / 20(3.8 \pm 0.6)$ & $0 / 20$ \\
& $4 / 17(1 \pm 0)$ & $2 / 17$ \\
\hline
\end{tabular}

$\mathrm{BALB} / \mathrm{c}$ mice were twice immunized with chromatographically purified myosin isoforms derived from rat or mouse hearts $(\alpha$-myosin) or soleus muscles ( $\beta$-myosin) at a weekly interval. Histopathology was determined 3 wk later. *Number of animals with soleus muscle myositis/animals immunized. ${ }^{\ddagger}$ Number of animals with myocarditis/animals imnmunized. ${ }^{\S}$ Severity was ranged from 1 to 4 (1 corresponds to infiltration of up to $5 \%$ of at least one histologic cross-section; $2,5-10 \% ; 3,10-20 \% ; 4,>20 \%$ ).

method of Shiverick et al. (14). The purity of the myosin preparations was further tested by SDS-PAGE (12). It should be noted that the soleus muscle does not exclusively contain $\beta$ myosin, but also minor amounts of II A- and II B-myosin (13). However, it has been shown that the II A- and II B-myosin isoforms do not cause myocarditis or myositis (5).

Synthesis of peptides derived from cardiac myosin. The polypeptides derived from cardiac myosin (see Table II) were synthesized by the Fmoc (fluorenylmethoxycarbonyl)/t-butyl based solid phase peptide chemistry method. An ABI 431 (Perkin Elmer Corp., Foster City, CA) peptide synthesizer using a single coupling program was used to carry out the chain assembly starting with commercially available Fmoc-AA-HMP (hydroxymethyl-phenylacetyl) derivatized polystyrene resin, where AA is the $\mathrm{COOH}$-terminal amino acid (aa) residue. Subsequent aa were coupled as hydroxybenztriazole esters. Upon removal of the final $\mathrm{NH}_{2}$-terminal Fmoc, side-chain protecting groups were removed and the peptides were cleaved from the resin by treatment for $4 \mathrm{~h}$ with TFA/thioanisole/ $\beta$-mercaptoethanol/water/ phenol $(80: 5: 5: 5: 5)$. The resulting suspension was filtered, and the filtrate volume was reduced by roto-evaporation. The crude peptide was precipitated and washed with ether, then dried in vacuo overnight. The acetylated polypeptides were also synthesized by Fmoc methodology. However, upon removal of the final $\mathrm{NH}_{2}$-terminal Fmoc, the $\mathrm{NH}_{2}$ terminus was acetylated by mixing the resin for 30 min in a solution of $25 \%$ acetic anhydride in dimethyl formamide. All peptides were purified by reverse-phase HPLC on a preparative $(2.5$ $\mathrm{cm} \times 25 \mathrm{~cm})$ Vydac $\mathrm{C}_{4}$ column with linear gradients of $99.95 \%$ acetonitrile plus $0.05 \%$ TFA vs. $0.1 \%$ aqueous TFA.

Immunization procedures. 6-8-wk-old BALB/c mice were immunized twice at a 7-d interval with purified cardiac or soleus muscle myosin emulsified in Freund's complete adjuvant (FCA $)^{1}$ as described (5). Peptides were diluted in PBS at a concentration of $1 \mathrm{mg} /$ $\mathrm{ml}$, emulsified in FCA, and injected according to the same protocol that was used for the full-length myosins. $3 \mathrm{wk}$ after the first injection, hearts, and both soleus muscles and quadriceps and biceps muscles were excised and fixed in formalin.

Histopathology. Sections from formalin-fixed, paraffin-embedded tissues were obtained at several levels and stained with hematoxylin and eosin. Myocarditis or myositis was determined by the presence of an inflammatory infiltrate and myocyte damage. The severity of myocarditis was determined according to a previously described scoring system (5) ranging from 1 to 4 (1 corresponds to infiltration of up to $5 \%$ of at least one histologic cross-section; $2,5-10 \% ; 3,10-20 \%$; $4>20 \%)$.

1. Abbreviations used in this paper: Ac, acetylated; FCA, Freund's complete adjuvant.

\section{Results}

$\alpha$ - and $\beta$-Myosin induce myocarditis at different prevalence and severity. To test whether $\alpha$ - and $\beta$-myosin obtained from mice and rats differ in their induction of myocarditis, BALB/c mice were immunized with these antigens. In agreement with previous findings (5), immunization with the murine cardiac myosin, i.e., $\alpha$-myosin, induced severe autoimmune myocarditis in the majority of mice. $\alpha$-Myosin obtained from rat hearts induced the disease at similar prevalence and severity (Table I).

In contrast, immunization with murine and rat $\beta$-myosin isoforms (obtained from soleus muscles) induced myocarditis at minimal prevalence and severity. In addition, $\beta$-myosin immunization also caused inflammation of the soleus muscle in a few animals. The inflammatory lesions induced by $\beta$-myosin were heart- and soleus muscle-specific, as other skeletal muscle tissues were normal upon histologic examination (data not shown).

The lesions in the heart tissue of $\beta$-myosin-immunized mice were similar to myocarditis induced by the $\alpha$-myosins but much less extended and characterized by a diffuse interstitial cellular infiltrate. The histologic picture of inflammation of the soleus muscle was similar (Fig. 1a). These data show that the murine and rat $\alpha$-myosin isoforms have the same pathogenicity and that $\alpha$-myosin is immunodominant in the pathogenesis of inflammatory heart disease.

Selection of candidate peptides derived from cardiac myosin heavy chain that potentially induce myocarditis. Since $\alpha$-myo$\sin$ but not $\beta$-myosin induces myocarditis at high prevalence and severity, we compared the amino acid sequence of the rat cardiac and soleus muscle myosin heavy chains. Peptides were synthesized from the rat $\alpha$-myosin regions that correspond to the clusters of difference. The rat $\alpha$ - and $\beta$-myosins had to be used for comparison because the amino acid sequence of mouse $\beta$-myosin is not known. However, the amino acid sequences of $\alpha$-myocins from rats and mice are almost identical (>99\%) (15) and both were found to be equally pathogenic (Table I). The differences between the rat $\alpha$ - and $\beta$-myosin heavy chain are restricted to 8 clusters of amino acids and to 52 single aa substitutions within a total length of 1938 and 1935 aa, respectively (16) (Fig. 2). In addition, the region encompassing aa 736-1032 was of particular interest because it had been shown recently that this region might contain pathogenic epitopes (17). Therefore, within this region we additionally synthesized short peptides corresponding to the rat $\alpha$-myosin 

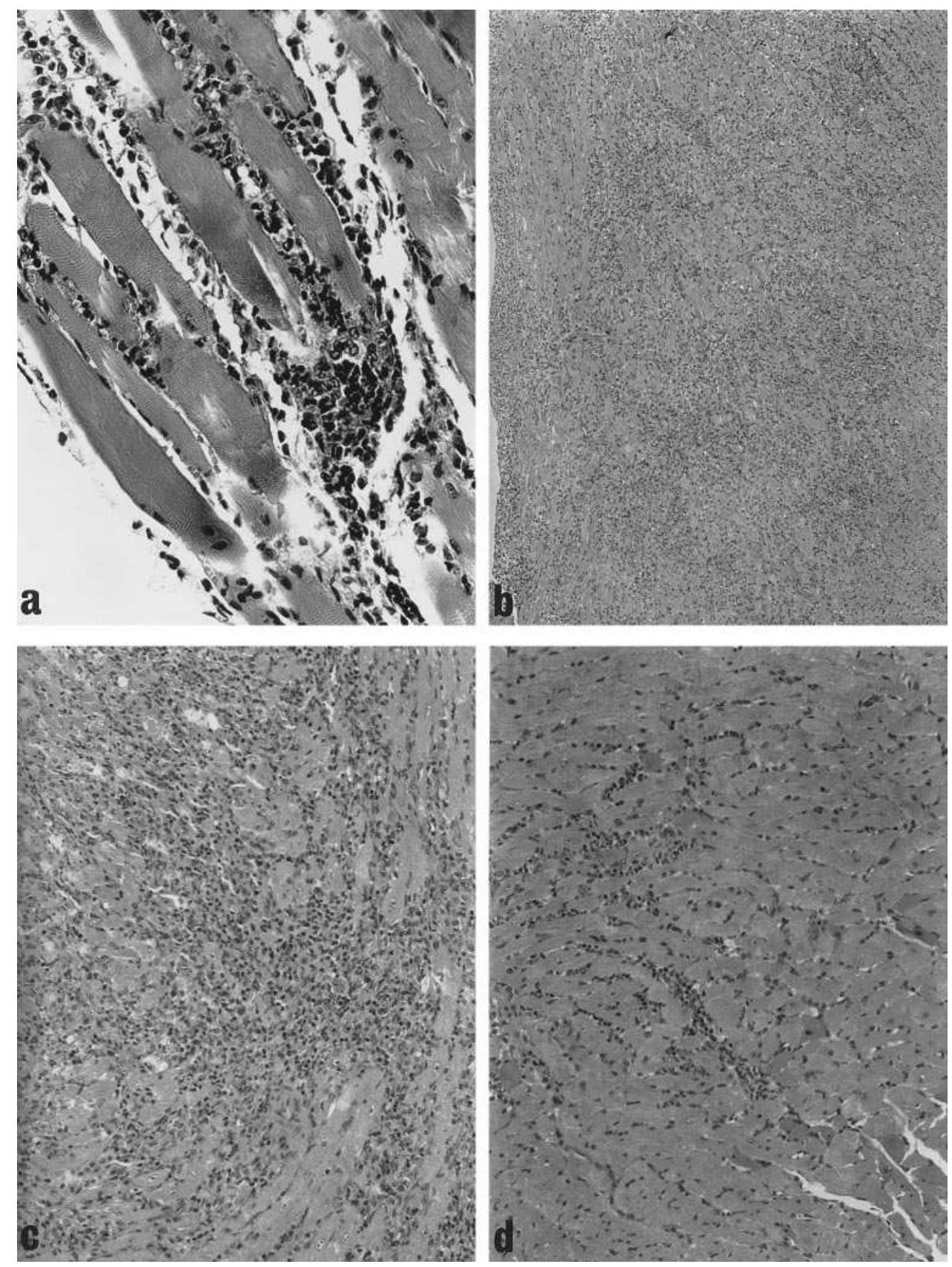

Figure 1. (a) Cellular infiltrate in the soleus muscle of a BALB/c mouse injected with rat soleus muscle myosin. The infiltrate of mononuclear cells is diffuse and interstitial. Hematoxylin and eosin staining. $\times 290$. (b) Extensive inflammatory infiltrate throughout the ventricular wall of a BALB/c mouse injected with the dominant pathogenic cardiac myosin peptide acetylated (Ac) aa 614-643. Hematoxylin and eosin staining. $\times 90$. (c) Same section as in $b$. The histology closely resembles that seen in cardiac myosin-immunized mice. $\times 180$. $(d)$ Representative histologic picture of the myocardium of a BALB/c mouse injected with the peptides with minor pathogenicity aa 735-747 and aa 947-960. The lesions in all cases were restricted to small inflammatory foci. $\times 180$.

that differed by only one aa from $\beta$-myosin. The peptide aa 1304-1320 was also included in this study because it had been shown to induce myocarditis in Lewis rats (18). Since acetylation of peptides has been described to alter their immunogenicity (19), peptides were also tested as $\mathrm{NH}_{2}$-terminally acetylated variants as well (Table II).

Identification of pathogenic $\alpha$-myosin peptides. Mice were immunized with the candidate peptides (Fig. 2) emulsified in FCA according to the immunization protocol used for the whole molecule. Among all peptides tested three were found to induce myocarditis (Table II). However, these three peptides significantly differed in their pathogenicity. The peptide aa 614-643 (cluster of difference) showed dominant pathogenicity and induced severe myocarditis with inflammatory lesions throughout the ventricular wall. (Fig. 1, $b$ and $c$ ). In contrast, myocarditis induced by the two other peptides corresponding to aa 735-747 and 947-960 was restricted to small foci of interstitial infiltration (Fig. $1 d$ ). $\mathrm{NH}_{2}$-terminal acetylation significantly changed the pathogenicity of the peptides. While the nonacetylated variant of the dominant peptide aa 614-643 failed to induce the disease, peptides aa 735-747 and 947-960 were only pathogenic in a nonacetylated state.

\section{Discussion}

In this report we have identified three peptides derived from the cardiac $\alpha$-myosin isoform that can induce autoimmune heart disease. The selection of peptides was based on our finding that myosin isoforms with similar amino acid sequence, i.e., cardiac and soleus muscle myosin, significantly differ in their ability to induce myocarditis.

Among the eight peptides corresponding to clusters of dif- 


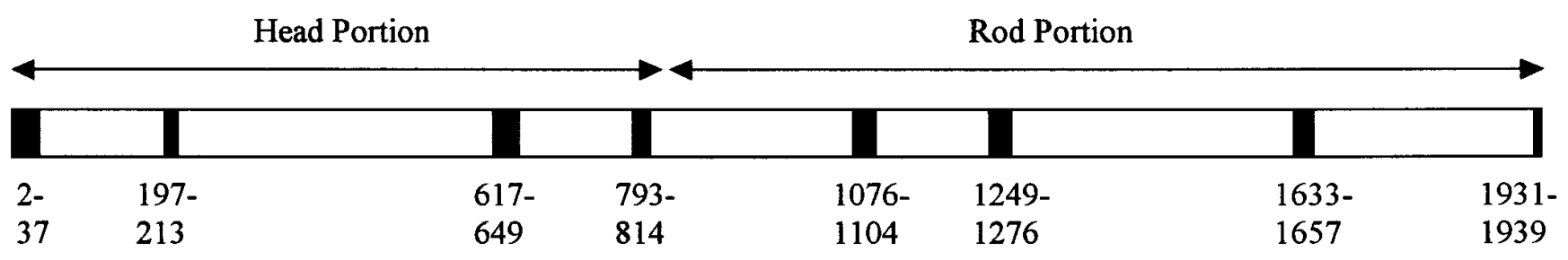

$\overline{100}$ aa

Figure 2. Schematic representation of the $\alpha$-myosin heavy chain. The black bars correspond to clusters of different amino acids when compared with the $\beta$-myosin heavy chain. The amino acid position of each cluster is indicated.

ferent amino acids between $\alpha$-myosin and $\beta$-myosin, only one showed pathogenicity. However, the pathogenicity of this peptide appeared to be dominant as it induced myocarditis at a severity comparable with immunization with the whole myosin molecule. The two other $\alpha$-myosin peptides displayed minor pathogenicity and were derived from regions that differed from $\beta$-myosin by only one amino acid, respectively. The immunodominant peptide differs from the corresponding $\beta$-myo-

Table II. Induction of Autoimmune Myocarditis by Peptides Corresponding to $\alpha$-Myosin

\begin{tabular}{|c|c|c|}
\hline$\alpha$-Myosin peptides (amino acid sequence)* & $\begin{array}{l}\text { Peptide position } \\
\text { amino acid No. }\end{array}$ & Myocarditis $^{\ddagger}$ (severity) \\
\hline MTDAQQMADEFGAAAㅍㄴRKSEKERL & $1-24$ & $0 / 5$ \\
\hline 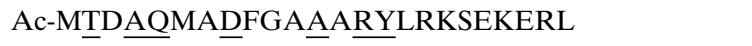 & & $0 / 5$ \\
\hline EAQTRPFDIRTECFVPDDKEEYYVKAKIVS & $25-53$ & $0 / 5$ \\
\hline Ac-EAQTRPFDIRTECFVPDDKEE $\underline{\bar{Y} V K A K I V S}$ & & $0 / 5$ \\
\hline SLKLMATLFSTYAㅅANㅁDSGKGKGGKKKG & $614-643$ & $0 / 10^{\S}$ \\
\hline 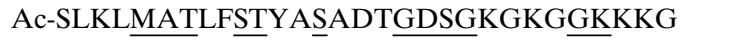 & & $8 / 22(3.5 \pm 0.7)^{\S}$ \\
\hline GQFIDSGKAGAEKL & $735-747$ & $3 / 12(1 \pm 0)^{\S}$ \\
\hline Ac-GQFIDSGKAGAEKL & & $0 / 5$ \\
\hline IQAQARRGQLLMRIEFKKIVERRDALLLVIQWN & $790-819$ & $0 / 12^{\S}$ \\
\hline 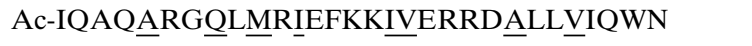 & & $0 / 5$ \\
\hline DECSELKKKDIDDLE & $947-960$ & $5 / 12(1 \pm 0)^{\S}$ \\
\hline Ac-DECSELKKKDIDDLE & & $0 / 5$ \\
\hline GLDEIIAKLTKEK & $986-998$ & $0 / 7$ \\
\hline Ac-GLDEIIĀKLTKEK & & $0 / 7$ \\
\hline KVNTLTKS్KVKLEQ & $1018-1031$ & $0 / 7$ \\
\hline Ac-KVNTLTKS̄KVKLEQ & & $0 / 6$ \\
\hline DKLQLEEEKLKKKEFFDISQQNSKIEDEQALALQLQ & $1074-1107$ & $0 / 5$ \\
\hline 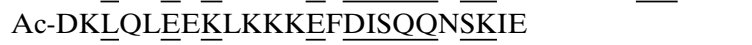 & $1074-1097$ & $0 / 8$ \\
\hline Ac-DEQALALQLQKKL & $1098-1110$ & $0 / 8$ \\
\hline 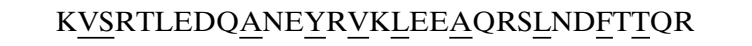 & $1249-1279$ & $0 / 5$ \\
\hline Ac-LEK $\underline{V}$ SRTLEDQ $\underline{\text { ANE }} \underline{\bar{Y}} \underline{R} \underline{\bar{V}}$ & $1247-1263$ & $0 / 5$ \\
\hline Ac- $\underline{Y} \underline{R} \underline{V} \underline{L} E E \underline{A} Q R \underline{L} \underline{N D} \underline{F T T Q R}$ & $1261-1279$ & $0 / 5$ \\
\hline Ac-TRGKLㅇYTQQMEEDLKRQ\| & $1304-1320$ & $0 / 5$ \\
\hline QLSQ QANRIASEAQKHLKNAQQAHLKDTQLQQLD & $1630-1660$ & $0 / 5$ \\
\hline 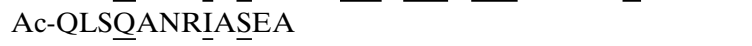 & $1630-1641$ & $0 / 5$ \\
\hline 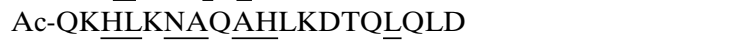 & $1642-1660$ & $0 / 5$ \\
\hline KLRAKSRDIGAKQKMHDEE & $1921-1939$ & $0 / 5$ \\
\hline Ac-RDIGAKQKMHDEE & $1927-1939$ & $0 / 5$ \\
\hline
\end{tabular}

$\mathrm{BALB} / \mathrm{c}$ mice were immunized twice at a weekly interval with synthetic peptides derived from cardiac $(\alpha-)$ myosin. Presence of myocarditis was determined 3 wk after the first immunization and the severity of the disease was ranged as described in Table I. * $(\mathrm{Ac}-)$ is $\mathrm{NH}_{2}$-terminally acetylated peptide. Amino acids that are underlined differ from the corresponding $\beta$-myosin sequence. ${ }^{*}$ Number of animals with myocarditis/number of animals immunized. "This peptide induces myocarditis in Lewis rats. ${ }^{\S}$ Pooled data from at least two experiments. 
sin region by 13 amino acids. This pronounced difference could account for its high pathogenicity, and conversely the minor activity of the two other peptides might be due to the fact that they differ by only one amino acid from the corresponding $\beta$-myosin region.

Immunization experiments in BALB/c mice with fragments of rat cardiac myosin suggested that pathogenic epitopes reside between aa 736 and 1032 (17). According to amino acid sequence comparison, this particular region contains five potentially myocarditogenic epitopes, namely one epitope with a cluster of difference and four epitopes that differ by a single amino acid from $\beta$-myosin. As demonstrated by the present study, the peptide spanning the cluster of difference was not pathogenic and among the four others we have identified two that showed minor pathogenicity. Thus, although we have identified two immunogenic peptides in the aa 736-1032 region, our data imply that the dominant pathogenic epitope is located outside this region, in the head portion of the molecule.

It has been shown recently that immunization with a peptide corresponding to aa 1304-1320 of the rat $\alpha$-myosin heavy chain induces myocarditis in Lewis rats (18). Since the amino acid sequence of this peptide is also found on the corresponding rat $\beta$-myosin region (16), we expected that this peptide might fail to induce the disease in $\mathrm{BALB} / \mathrm{c}$ mice.

In a very recent report it has been demonstrated that a peptide corresponding to aa 334-352 can induce myocarditis in A/J mice and specifically binds to class II MHC molecules (I-A ${ }^{k}$ ) of $\mathrm{A} / \mathrm{J}$ mice (20). In our study a peptide spanning this particular region failed to induce the disease in $\mathrm{BALB} / \mathrm{c}$ mice (class II MHC I-A ${ }^{\mathrm{d}}$, data not shown). Therefore it is likely that strainspecific differences in the response to $\alpha$-myosin are associated with specific differences in class II MHC haplotypes. In addition, with the great length of $\alpha$-myosin it would be surprising if the pathogenicity of this antigen was restricted to only one epitope, particularly in different strains of mice. The fact that the aa 334-352 peptide differs from the corresponding region of $\beta$-myosin by five amino acids underscores our hypothesis that dominant epitopes are located in regions of difference between $\alpha$ - and $\beta$-myosin isoforms.

$\mathrm{NH}_{2}$-terminal acetylation of peptides derived from self antigens was reported to enhance their antigenicity in some cases (19). The mechanism by which acetylation influences immunogenicity is not clear but may be based on $(a)$ the prevention of extra- or intracellular peptide degradation $(b)$ enhanced peptide binding to MHC class II molecules, or $(c)$ targeting peptides to specific intracellular compartments for MHC class II association (19). The effect of acetylation is most evident in peptide aa 614-643 because lack of the acetyl moiety completely abrogates its pathogenic potential. $\mathrm{NH}_{2}$-terminal acetylation of the pathogenic peptide was required in the Lewis rat model as well (18). Conversely, the peptides with minor pathogenicity lose their antigenicity when acetylated.

Immunization with $\beta$-myosin induced both myocarditis and soleus muscle myositis at minimal prevalence and severity. Thus, there must be a partial cross-reactivity between $\alpha$ - and $\beta$-myosin isoforms at the $\mathrm{T}$ cell level. The cross-reactive epitopes could not be delineated with our approach but must possess minor pathogenicity. Most probably they are located within regions that are identical in the $\alpha$ - and $\beta$-myosin isoforms. The minimal prevalence of soleus muscle inflammation could be due to $(a)$ lack of relevant $\beta$-myosin-specific $\mathrm{T}$ cell clones and/or $(b)$ less pronounced target organ susceptibility.
The first hypothesis may be explained by immuonlogic tolerance to $\beta$-myosin which, in contrast to the $\alpha$-isoform, is already expressed during embryonic development (21). The second hypothesis is supported by the observation that skeletal muscle tissues including soleus contain much less cytokine-inducible antigen presenting cells as compared with the heart (reference 22 and our unpublished data).

Soleus myositis is the first example of myositis induced by a defined autoantigen, i.e., $\beta$-myosin. This autoantigen also constitutes a link between myositis and myocarditis because it induced both myocarditis and myositis. In patients with myocarditis and dilated cardiomyopathy it has been suggested that the $\alpha$ - and $\beta$-myosin heavy chains could be important autoantigens (23). The human $\alpha$-myosin heavy chain is heart specific and mostly found in the atrium, whereas the $\beta$-chain is mainly found in the ventricle, but also in slow skeletal muscles (8). Thus, it is conceivable that in myocarditis patients an autoimmune response to the $\beta$-myosin heavy chain may also affect slow skeletal muscles. In addition, autoimmune responses against skeletal muscle with $\beta$-myosin may lead to heart disease.

The identification of pathogenic epitopes on cardiac myo$\sin$ is an important step to further dissect the molecular mechanisms acting in ongoing heart disease. It is conceivable that peptides with slightly modified amino acid sequence can be applied to antagonize the original pathogenic epitope (24) and subsequently modulate ongoing heart disease. Altered peptide ligands were successfully used to downmodulate the disease in experimental encophalomyelitis (25) and adjuvant arthritis (26). Studies are currently underway to determine whether this approach will also be useful in autoimmune myocarditis.

\section{Acknowledgments}

The excellent technical assistance of S. Joebstel and M. Lorenz is gratefully acknowledged.

This work was supported by a grant from the Austrian Fonds zür Foerderung der Wissenschaftlichen Forschung (8960).

\section{References}

1. Woodruff, J.F. 1980. Viral myocarditis: a review. Am. J. Pathol. 101:427484.

2. Olinde, K.B., and J.B. O'Connel. 1994. Inflammatory heart disease: pathogenesis, clinical manifestations, and treatment of myocarditis. Annu. Rev. Med. 45:481-490.

3. James, Th.N. 1983. Myocarditis and cardiomyopathy. N. Engl. J. Med. 308:39-42.

4. Huber, S.A. 1992. Viral myocarditis: a tale of two diseases. Lab. Invest. 66:1-3.

5. Neu, N., N.R. Rose, K.W. Beisel, A. Herskowitz, G. Gurri-Glass, and S.W. Craig. 1987. Cardiac myosin induces myocarditis in genetically predisposed mice. J. Immunol. 139:3630-3636.

6. Smith, S.C., and P.M. Allen. 1991. Myosin-induced acute myocarditis is a T cell-mediated disease. J. Immunol. 147:2141-2147.

7. Pummerer, C., P. Berger, M. Frühwirth, C. Öfner, and N. Neu. 1991. Cellular infiltrate, major histocompatibility antigen expression and immunopathogenic mechanisms in cardiac myosin-induced myocarditis. Lab. Invest. 65:538547.

8. Sartorelli, V., M. Kurabayashi, and L. Kedes. 1993. Muscle-specific gene expression. A comparison of cardiac and skeletal muscle transcription strategies. Circ. Res. 72:925-931.

9. Morkin, E. 1993. Regulation of myosin heavy chain genes in the heart Circulation. 87:1451-1460.

10. Nadal-Ginard, B., and V. Mahdavi. 1989. Molecular basis of cardiac performance. J. Clin. Invest. 84:1693-1700.

11. Emerson, C.P., and S.I. Bernstein. 1987. Molecular genetics of myosin. Ann. Rev. Biochem. 56:695-726.

12. Neu, N., S.W. Craig, N.R. Rose, F. Alvarez, and K.W. Beisel. 1987. Coxsackievirus induced myocarditis in mice: cardiac myosin autoantibodies do not 
cross-react with the virus. Clin. Exp. Immunol. 69:566-574.

13. Izumo, S., B. Nadal-Ginard, and V. Mahdavi. 1986. All members of the myosin heavy chain multi-gene family respond to thyroid hormone in a highly tissue specific manner. Science (Wash. DC). 231:597-600.

14. Shiverick, K.T., L.L. Thomas, and N.R. Alpert. 1975. Purification of cardiac myosin. Application to hypertrophied myocardium. Biochim. Biophys. Acta. 393:124-129.

15. Quinn-Laquer, B.K., J.E. Kennedy, S.J. Wei, and K.W. Beisel. 1992. Characterisation of the allelic differences in the mouse cardiac $\alpha$-myosin heavy chain coding sequence. Genomics. 13:176-188.

16. McNally, E.M., R. Kraft, M. Bravo-Zehnder, D.A. Tayolor, and L.A. Leinwand. 1989. Full-length rat alpha and beta cardiac myosin heavy chain sequences: comparison suggests a molecular basis for function differences. J. Mol. Biol. 210:655-671.

17. Liao, L., R. Sindhwani, L. Leinward, B. Diamond, and S. Factor. 1993. Cardiac $\alpha$-myosin heavy chains differ in their induction of myocarditis. Identification of pathogenic epitopes. J. Clin. Invest. 90:2877-2882.

18. Wegmann, K.W., W. Zhao, A.C. Griffin, and W.F. Hickey 1994. Identification of myocarditogenic peptides derived from cardiac myosin capable of inducing experimental allergic myocarditis in the Lewis rat. J. Immunol. 153:892900

19. Zhao, W., K.W. Wegmann, J.L. Trotter, K. Ueno, and W.F. Hickey. 1994. Identification of an N-terminally acetylated encephaltogenic epitope in myelin proteolipid apoprotein for Lewis rat. J. Immunol. 153:901-909.
20. Donermeyer, D.L., K.W. Beisel, P.M. Allen, and S.C. Smith. 1995. Myocarditis-inducing epitope of myosin binds constitutively and stably to I-A ${ }^{\mathrm{k}}$ on antigen-presenting cells in the heart. J. Exp. Med. 182:1291-1300.

21. Schiffiano, S., and C. Reggiani. 1994. Myosin isoforms in mammalian skeletal muscle. J. Appl. Physiol. 77:493-501.

22. Smith, S.C., and P.M. Allen. 1992. Expression of myosin-class II major histocompatibility complexes in the normal myocardium occurs before induction of autoimmune myocarditis. Proc. Natl. Acad. Sci. USA. 89:9131-9135.

23. Caforio, A.L.P., M. Grazzini, J.M. Mann, P.J. Keeling, G.F. Bottazzo, W.J. McKenna, and S. Schiffano. 1992. Identification of $\alpha$ - and $\beta$-cardiac myosin heavy chain isoforms as major autoantigens in dilated cardiomyopathy. Circulation. 85:1734-1742.

24. Sloan-Lancaster, J., and P.M. Allen. 1995. Significance of T-cell stimulation by altered peptide ligands in T cell biology. Curr. Opin. Immunol. 7:103109.

25. Franco, A., S. Southwood, T. Arrhenius, V.K. Kuchroo, H.M. Grey, A. Sette, and G.Y. Ishioka. 1994. T cell receptor antagonist peptides are highly effective inhibitors of experimental encephalomyelitis. Eur. J. Immunol. 24:940946.

26. Wauben, M.H.M., C.J.P. Boog, R. Van der Zee, I. Joosten, A. Schleif, and W. Van Eden. 1992. Disease inhibition by major histocompatibility complex binding peptide analogues or disease-associated epitopes: more than blocking alone. J. Exp. Med. 176:667-677. 